H. Yokoi

Nagoya Math. J.

Vol. 120 (1990), 51-59

\title{
THE FUNDAMENTAL UNIT AND CLASS NUMBER ONE PROBLEM OF REAL QUADRATIC FIELDS WITH PRIME DISCRIMINANT
}

\author{
HIDEO YOKOI
}

\section{§ 0 . Introduction}

Class number one problem for imaginary quadratic fields was solved in 1966 by A. Baker and H.M. Stark independently. However, the problem for real quadratic fields is still unsolved. It seems to us that one of the most essential difficulties of the problem for real quadratic fields comes from deep connection of the class number with the fundamental unit.

In this paper, we shall first in $\S 1$ concern ourselves with real quadratic fields of prime discriminant $F=\boldsymbol{Q}(\sqrt{p})($ prime $p \equiv 1 \bmod 4)$, and give a sufficient condition for an unit $\varepsilon=(t+u \sqrt{p}) / 2$ corresponding to a positive integral solution $(x, y)=(t, u)$ of the diophantine equation $x^{2}-p y^{2}=-4$ to be the fundamental unit (Theorem 1 ).

In $\S 2$, for the $p$-invariant $n_{p}$ defined by using the fundamental unit of $F$

$$
\varepsilon_{p}=\left(t_{p}+u_{p} \sqrt{p}\right) / 2 \quad(>1)
$$

in the case $n_{p} \neq 0$, i.e. $t_{p} / u_{p}^{2}>1 / 2$, the class number one problem is considered, and it will be proved that if $n_{p} \neq 0$ and $h_{p}=1$ then $p<4.1 \times 10^{6}$ holds with one possible exception of $p$ and that under the assumption of the generalized Riemann hypothesis this is true without any exception (Theorem 2).

Finally, we shall show that for real quadratic fields $\boldsymbol{Q}(\sqrt{ } \bar{d})$ with discriminant $d$ not necessarily prime the same result is proved (Theorem 3 ). Moreover, we shall give three kinds of tables, one of which consists of 30 primes $p$ congruent to $1 \bmod 4$ satisfying $h_{p}=1$ and $n_{p} \geqq 1$.

\section{§1. Fundamental unit}

In real quadratic fields $\boldsymbol{Q}(\sqrt{p})$ with prime discriminant, $p$ is prime Received November 17, 1989. 
congruent to $1 \bmod 4$. Therefore, the fundamental unit of $\boldsymbol{Q}(\sqrt{p})$

$$
\varepsilon_{p}=\left(t_{p}+u_{p} \sqrt{p}\right) / 2 \quad(>1),
$$

has the norm $N \varepsilon_{p}=-1$, and hence $\left(t_{p}, u_{p}\right)$ is the smallest positive integral solution of $x^{2}-p y^{2}=-4$.

Conversely, we can prove the following theorem:

Theorem 1. For any fixed prime $p(\neq 5)$ congruent to $1 \bmod 4$, if a non-trivial positive integral solution $(x, y)=(t, u)(t>0, u>0)$ of diophantine equation $x^{2}-p y^{2}=-4$ satisfies any one of the following:

(i) $t / u^{2}>1 / 2$,

(ii) $t<2 p$,

(iii) $u^{2}<4 p$,

then $\varepsilon=(t+u \sqrt{p}) / 2$ is the fundamental unit of the real quadratic field $\boldsymbol{Q}(\sqrt{p})$.

To prove this theorem, we need several lemmas. First we prove the following:

Lemma 1. For any prime $p$ congruent to $1 \bmod 4$, let $(t, u)$ be a positive integral solution of diophantine equation $x^{2}-p y^{2}=-4$.

Then the following are equivalent:

(i) $t / u^{2}>1 / 2$,

(ii) $t<2 p$,

(iii) $u^{2}<4 p$.

Proof. In the case $u=1$, we have $p=t^{2}+4$, and hence $t=\sqrt{p-4}$ $<2 p, u^{2}=1<4 p, t / u^{2} \geqq 1$.

In the case $u=2$, we have $p=t^{2} / 4+1$, and hence $t=2 \sqrt{p-1}<2 p$, $u^{2}=4<4 p, t / u^{2} \geqq 1$.

Now we suppose $u>2$. Then $t^{2}-p u^{2}=-4$ implies $t \neq 2 p, 0<8 / u^{2}$ $<1$ and $p>p-4 / u^{2}=t^{2} / u^{2}$. Therefore,

$$
t / u^{2}<1 / 2 \quad \text { if and only if } \quad p-4 / u^{2}<t / 2,
$$

which is equivalent to $t>2 p$.

Next, $p \geqq 5$ implies $0<4 / p<1$. Therefore,

$$
t>2 p \quad \text { if and only if } \quad p u^{2}-4>4 p^{2},
$$

which is equivalent to $u^{2}>4 p$. 
Lemma 2. For any prime $p$ congruent to $1 \bmod 4$, let $(x, y)=\left(t_{0}, u_{0}\right)$ be any positive integral solution of diophantine equation $x^{2}-p y^{2}=-4$. If we put

$$
\left(\frac{t_{0}+u_{0} \sqrt{p}}{2}\right)^{2 n+1}=\frac{t_{n}+u_{n} \sqrt{p}}{2} \quad(n=1,2, \cdots),
$$

then sequences of natural numbers $\left\{t_{n}\right\},\left\{u_{n}\right\}$ are monotonically increasing in narrow sense.

Proof. If we put

$$
\frac{t+u \sqrt{p}}{2}=\left(\frac{t_{0}+u_{0} \sqrt{p}}{2}\right)^{2}
$$

then we get easily

$$
t=\left(t_{0}^{2}+p u_{0}^{2}\right) / 2 \geqq 3,
$$

and hence we have $t / 2>1$.

On the other hand, from the definition, we have

$$
\begin{aligned}
\frac{t_{n+1}+u_{n+1} \sqrt{p}}{2} & =\frac{t_{n}+u_{n} \sqrt{p}}{2} \cdot \frac{t+u \sqrt{p}}{2} \\
& =\left\{\left(t t_{n}+p u u_{n}\right)+\left(t u_{n}+u t_{n}\right) \sqrt{p}\right\} / 4 .
\end{aligned}
$$

Therefore, we get

$$
t_{n+1}=(t / 2) t_{n}+(p / 2) u u_{n}>t_{n}
$$

and

$$
u_{n+1}=(t / 2) u_{n}+(u / 2) t_{n}>u_{n}
$$

for any $n=1,2,3, \cdots$.

LEMMA 3. For any prime $p$ congruent to $1 \bmod 4$, let

$$
\varepsilon_{p}=\left(t_{p}+u_{p} \sqrt{p}\right) / 2 \quad(>1)
$$

be the fundamental unit of real quadratic field $\boldsymbol{Q}(\sqrt{\bar{p}})$. If we put

$$
\varepsilon_{p}^{3}=\left(\bar{t}_{p}+\bar{u}_{p} \sqrt{p}\right) / 2,
$$

then

$$
\bar{t}_{p}>2 p \quad \text { and } \quad \bar{u}_{p}^{2}>4 p
$$

hold except for $p=5$. 
Proof. Since

$$
\begin{aligned}
\left(\bar{t}_{p}+\bar{u}_{p} \sqrt{p}\right) / 2 & =\left\{\left(t_{p}+u_{p} \sqrt{p}\right) / 2\right\}^{3} \\
& =\left[\left\{\left(t_{p}^{3}+3 p t_{p} u_{p}^{2}\right)+\left(p u_{p}^{3}+3 t_{p}^{2} u_{p}\right) \sqrt{p}\right\} / 4\right] / 2 .
\end{aligned}
$$

we have

$$
\bar{t}_{p}=t_{p}\left(t_{p}^{2}+3 p u_{p}^{2}\right) / 4
$$

and

$$
\bar{u}_{p}=u_{p}\left(p u_{p}^{2}+3 t_{p}^{2}\right) / 4 \text {. }
$$

Hence, $2 p<\bar{t}_{p}$ holds if and only if

$$
p\left(8-3 t_{p} u_{p}^{2}\right)<t_{p}^{3}
$$

which follows from

$$
8-3 t_{p} u_{p}^{2}<0, \quad \text { i.e. } \quad u_{p}^{2} t_{p} \geqq 3 .
$$

However, $u_{p}^{2} t_{p} \geqq 3$ holds if and only if $u_{p} \neq 1$ or $t_{p} \neq 1$, which is equivalent to $p \neq 5$.

On the other hand, since $\left(\bar{t}_{p}, \bar{u}_{p}\right)$ is a positive integral solution of $x^{2}-p y^{2}=-4$, by Lemma 1

$$
\bar{t}_{p}>2 p \quad \text { if and only if } \quad \bar{u}_{p}^{2}>4 p .
$$

Proof of Theorem 1. First, we note that three conditions of our theorem are equivalent by Lemma 1 .

Next, we suppose that $\varepsilon=(t+u \sqrt{p}) / 2$ is not equal to the fundamental unit $\varepsilon_{p}=\left(t_{p}+u_{p} \sqrt{p}\right) / 2(>1)$ of $\boldsymbol{Q}(\sqrt{p})$, and put

$$
\left(\frac{t_{p}+u_{p} \sqrt{p}}{2}\right)^{2 n+1}=\frac{t_{n}+u_{n} \sqrt{p}}{2} \quad(n=1,2, \cdots) .
$$

Then there is an uniquely determined positive integer $m$ such that

$$
t=t_{m} \quad \text { and } \quad u=u_{m},
$$

and by Lemma 2, we have

$$
t_{p}<t_{1} \leqq t_{m} \quad \text { and } \quad u_{p}<u_{1} \leqq u_{m} .
$$

On the other hand, by Lemma 3, we obtain

$$
t_{1}>2 p \quad \text { and } \quad u_{1}^{2}>4 p,
$$

which contradict with the assumption of our theorem. 


\section{§ 2. Class number}

For any prime $p$ congruent to $1 \bmod 4$, denote by

$$
\varepsilon_{p}=\left(t_{p}+u_{p} \sqrt{p}\right) / 2 \quad(>1)
$$

the fundamental unit of the real quadratic field $\boldsymbol{Q}(\sqrt{p})$ with prime discriminant.

We now define p-invariant as a mapping from the set of all rational primes congruent to $1 \bmod 4$ to the set of non-negative rational integers.

In our recent papers ([6], [7], [8]), we defined some new $p$-invariant, above all $n_{p}$, which is defined by the inequality

$$
\left|t_{p}\right| u_{p}^{2}-n_{p} \mid<1 / 2
$$

and obtained several interesting results regarding its property. Especially as a result closely related to class number one problem, we proved in [8] that in the case $n_{p} \neq 0$, there exists only a finite number of prime $p$ congruent to $1 \bmod 4$ with class number one.

In this section, we prove more precisely the following:

TheOREM 2. If prime $p$ congruent to $1 \bmod 4$ satisfies

$$
p>4.1 \times 10^{8} \quad \text { and } \quad n_{p} \neq 0 \quad \text { i.e. } \quad \varepsilon_{p}<2 p,
$$

then $h_{p}>1$ holds with one possible exception of $p$.

Moreover, if we assume the generalized Riemann Hypothesis, this is true without any exception.

The proof of this theorem depends upon the following lemma:

LEMMA 4. If prime $p$ congruent to $1 \bmod 4$ satisfies $n_{p} \neq 0$, then

$$
\varepsilon_{p}<2 p \quad \text { and } \quad h_{p}>\frac{0.3275}{m} \times \frac{p^{(m-2) / 2 m}}{\log 2 p}
$$

hold for any $p>e^{m}, m \geqq 11.2$ with one possible exception of $p$.

Assuming the generalized Riemann Hypothesis, this is true without any exception.

Proof. The first part of this lemma follows from Dirichlet's class number formula by applying the Siegel-Tatuzawa theorem (cf. [1], [5]) and Lemma 1.

For the second part, Kim [3] shows that if we assume the generalized 
Riemann Hypothesis, the Siegel-Tatuzawa theorem is true without any exception (cf. [4]).

Proof of Theorem. Put

$$
f_{m}(x)=\frac{x^{a}}{\log 2 x}, \quad a=a(m)=(m-2) / 2 m \quad(>0)
$$

for any fixed $m \geqq 11.2$. Then, since

$$
f_{m}^{\prime}(x)=\frac{(a \log 2 x)-1}{x^{1-a}(\log 2 x)^{2}}>0
$$

for any $x \geqq 6, f(x)$ is increasing on $[6, \infty)$.

Moreover, put

$$
g_{m}(x)=\frac{0.3275}{m} \times f_{m}(x)
$$

Then, for $m=15(>11.2)$ we have

$$
g_{15}\left(4.1 \times 10^{6}\right)>1, \quad \text { and } \quad e^{m}=e^{15}<4.1 \times 10^{6} .
$$

This establishes by Lemma 4 that $h_{p}>1$ holds for all $p>4.1 \times 10^{6}$ except possibly one $p$, and without any exception under the assumption of the generalized Riemann Hypothesis.

For any prime $p$ satisfying $3533<p<4.1 \times 10^{6}$, we may confirm that $n_{p} \neq 0$ implies $h_{p}>1$ by using Kida's UBASIC 86. We owe to Y. Tanigawa such better upper bound of $p$ and this confirmation. Moreover, in primes $p$ satisfying $5 \leqq p \leqq 3533$, we find exactly 30 primes $p$ such that $n_{p} \neq 0$ and $h_{p}=1$. Therefore, from Theorem 2 we obtain the following corollary, which is a generalization of Kim, Leu and Ono's result (cf. [2]):

Corollary. There exist exactly 30 primes $p$ congruent to $1 \bmod 4$ satisfying $n_{p} \geqq 1$ and $h_{p}=1$ with one more possible exception of $p$.

All such primes are listed in the following table I. Furthermore, Y. Tanigawa kindly informed me that by the same way the following general result is proved for discriminant $d$, not necessarily prime:

THEOREM 3. There exist exactly 54 discriminants $d$ of real quadratic fields $\boldsymbol{Q}(\sqrt{d})$ satisfying $\varepsilon_{d}<2 d$ and $h_{d}=1$ with one more possible exception of $d$.

All such discriminants except primes are listed in the table III. 
Table I

$\left(n_{p} \geqq 1, h_{p}=1\right)$

\begin{tabular}{|r|r|r|r|}
\hline \multicolumn{1}{|c|}{$p$} & \multicolumn{1}{|c|}{$t_{p}$} & $u_{p}$ & $n_{p}$ \\
\hline 5 & 1 & 1 & 1 \\
13 & 3 & 1 & 3 \\
17 & 8 & 2 & 2 \\
29 & 5 & 1 & 5 \\
37 & 12 & 2 & 3 \\
41 & 64 & 10 & 1 \\
53 & 7 & 1 & 7 \\
61 & 39 & 5 & 2 \\
101 & 20 & 2 & 5 \\
149 & 61 & 5 & 2 \\
157 & 213 & 17 & 1 \\
173 & 13 & 1 & 13 \\
197 & 28 & 2 & 7 \\
269 & 164 & 10 & 2 \\
293 & 17 & 1 & 17 \\
317 & 89 & 5 & 4 \\
461 & 365 & 17 & 1 \\
509 & 925 & 41 & 1 \\
557 & 236 & 10 & 2 \\
677 & 52 & 2 & 13 \\
773 & 139 & 5 & 6 \\
797 & 367 & 13 & 2 \\
941 & 1135 & 37 & 1 \\
1013 & 923 & 29 & 1 \\
1493 & 2357 & 61 & 1 \\
1613 & 2972 & 74 & 1 \\
1877 & 1603 & 37 & 1 \\
2477 & 647 & 13 & 4 \\
2693 & 4411 & 85 & 1 \\
3533 & 2437 & 41 & 1 \\
& & & \\
\hline & & \\
\hline
\end{tabular}

Table II

$\left(t_{p} / u_{p}^{2}>1 / 2, h_{p}>1\right)$

\begin{tabular}{|r|r|r|c|r|}
\hline$p$ & \multicolumn{1}{|c|}{$t_{p}$} & $u_{p}$ & $t_{p} / u_{p}^{2}$ & $h_{p}$ \\
\hline 229 & 15 & 1 & 15 & 3 \\
257 & 32 & 2 & 8 & 3 \\
401 & 40 & 2 & 10 & 5 \\
577 & 48 & 2 & 12 & 7 \\
733 & 27 & 1 & 27 & 3 \\
1009 & 1080 & 34 & 0.93 & 7 \\
1093 & 33 & 1 & 33 & 5 \\
1129 & 336 & 10 & 3.36 & 9 \\
1229 & 35 & 1 & 35 & 3 \\
1297 & 72 & 2 & 18 & 11 \\
1373 & 37 & 1 & 37 & 3 \\
1429 & 189 & 5 & 7.56 & 5 \\
1601 & 80 & 2 & 20 & 7 \\
1901 & 436 & 10 & 4.36 & 3 \\
2029 & 45 & 1 & 45 & 7 \\
2153 & 464 & 10 & 4.64 & 5 \\
2213 & 47 & 1 & 47 & 3 \\
2677 & 3777 & 73 & 0.70 & 3 \\
2917 & 108 & 2 & 27 & 3 \\
3137 & 112 & 2 & 28 & 9 \\
3181 & 564 & 10 & 5.64 & 5 \\
3221 & 3689 & 65 & 0.87 & 3 \\
3253 & 57 & 1 & 57 & 5 \\
4229 & 65 & 1 & 65 & 7 \\
4357 & 132 & 2 & 33 & 5 \\
4409 & 664 & 10 & 6.64 & 9 \\
4493 & 67 & 1 & 67 & 3 \\
4597 & 339 & 5 & 13.5 & 3 \\
4933 & 2388 & 34 & 2 & 3 \\
5273 & 1888 & 26 & 3 & 7 \\
\hline & & & & \\
\hline
\end{tabular}

$p$ : prime congruent to $1 \bmod 4$.

$\varepsilon_{p}=\left(t_{p}+u_{p} \sqrt{p}\right)>1$ : fundamental unit of $\boldsymbol{Q}(\sqrt{ } \bar{p})$.

$n_{p}: \quad p$-invariant defined by $\left|t_{p} / u_{p}^{2}-n_{p}\right|<1 / 2$.

$h_{p}$ : class number of $\boldsymbol{Q}(\sqrt{p})$. 
Table III

$$
\left(d \neq p, t_{d} / u_{d}^{2}>1 / 2 \text { i.e. } \quad n_{d} \geqq 1, h_{d}=1\right)
$$

\begin{tabular}{|c|c|c|c|c|}
\hline$d$ & $t_{d}$ & $u_{d}$ & $t_{d} / u_{d}^{2}$ & $n_{d}$ \\
\hline $21=3 \cdot 7$ & 5 & 1 & 5 & 5 \\
\hline $33=3 \cdot 11$ & 46 & 8 & 0.71 & 1 \\
\hline $69=3 \cdot 23$ & 25 & 3 & 2.77 & 3 \\
\hline $77=7 \cdot 11$ & 9 & 1 & 9 & 9 \\
\hline $93=3 \cdot 31$ & 29 & 3 & 3.20 & 3 \\
\hline $133=7 \cdot 19$ & 173 & 15 & 0.76 & 1 \\
\hline $141=3 \cdot 47$ & 190 & 16 & 0.74 & 1 \\
\hline $213=3 \cdot 71$ & 73 & 5 & 2.92 & 3 \\
\hline $237=3 \cdot 79$ & 77 & 5 & 3.08 & 3 \\
\hline $341=11 \cdot 31$ & 277 & 15 & 1.23 & 1 \\
\hline $413=7 \cdot 59$ & 61 & 3 & 6.77 & 7 \\
\hline $437=19 \cdot 23$ & 21 & 1 & 21 & 21 \\
\hline $453=3 \cdot 151$ & 149 & 7 & 3. 04 & 3 \\
\hline $573=3 \cdot 191$ & 766 & 32 & 0.74 & 1 \\
\hline $717=3 \cdot 239$ & 241 & 9 & 2.97 & 3 \\
\hline $917=7 \cdot 131$ & 1181 & 39 & 0.77 & 1 \\
\hline $1077=3 \cdot 359$ & 361 & 11 & 2.98 & 3 \\
\hline $1133=11 \cdot 103$ & 101 & 3 & 11.22 & 11 \\
\hline $1253=7 \cdot 179$ & 177 & 5 & 7.08 & 7 \\
\hline $1293=3 \cdot 431$ & 1726 & 48 & 0.74 & 1 \\
\hline $1757=7 \cdot 251$ & 1006 & 24 & 1.74 & 2 \\
\hline $2453=11 \cdot 223$ & 3566 & 72 & 0.68 & 1 \\
\hline $3053=43 \cdot 71$ & 3481 & 63 & 0.87 & 1 \\
\hline $3317=31 \cdot 107$ & 5241 & 91 & 0.63 & 1 \\
\hline
\end{tabular}

$d$ : discriminant of real quadratic $\boldsymbol{Q}(\sqrt{d})$.

$\varepsilon_{d}=\left(t_{d}+u_{d} \sqrt{d}\right) / 2>1$ : fundamental unit of $\boldsymbol{Q}(\sqrt{d})$.

$n_{d}$ : invariandt defined by $\left|t_{d} / n_{d}^{2}-n_{d}\right|<1 / 2$.

$h_{d}$ : class number of $\boldsymbol{Q}(\sqrt{ } \bar{d})$. 


\section{REFERENCES}

[1] J. Hoffstein, On the Siegel-Tatuzawa theorem, Acta Arith., 38 (1980), 167-174.

[2] H. K. Kim, M.-G. Leu and T. Ono, On two conjectures on real quadratic fields, Proc. Japan Acad., 63 (1987), 222-224.

[ 3 ] H. K. Kim, A conjecture of S. Chowla and related topics in analytic number theory, thesis, Johns Hopkins University, 1988.

[4] R. A. Mollin and H. C. Williams, A conjecture of S. Chowla via the generalized Riemann hypothesis, Proc. A. M. S., 102 (1988), 794-796.

[ 5 ] T. Tatuzawa, On a theorem of Siegel, Japan. J. Math., 21 (1951), 163-178.

[6] H. Yokoi, Some relations among new invariants of prime number $p$ congruent to 1 mod 4, Advances in pure Math., 13 (1988), 493-501.

[7] - New invariants of real quadratic fields, Proc. 1st Conf. Canadian Number Theory Assoc., April, 18-27, 1988, Banff, Canada, 635-639.

[8] - Bounds for fundamental units and class numbers of real quadratic fields with prime discriminant, Dept. of Math., Coll. Gen. Educ., Nagoya Univ., Prep. ser., 1989 , No. 2.

Department of Mathematics

College of General Education

Nagoya University

Chikusa-ku, Nagoya 464-01

Japan 